\title{
The correlation between the abundance of EGFR T790M mutation and osimertinib response in advanced non-small cell lung cancer
}

\author{
Guoqiang Pan ${ }^{1,2,3} \wedge$, Kaiyan Chen ${ }^{2,3}$, Xiaoqing Yu ${ }^{2,3}$, Jiamin Sheng ${ }^{1,2,3}$, Yun Fan ${ }^{1,2,3}$ \\ ${ }^{1}$ The First Clinical Medical College of Wenzhou Medical University, Wenzhou, China; ${ }^{2}$ The Cancer Hospital of the University of Chinese Academy \\ of Sciences (Zhejiang Cancer Hospital), Hangzhou, China; ${ }^{3}$ Institute of Basic Medicine and Cancer (IBMC), Chinese Academy of Sciences, \\ Hangzhou, China \\ Contributions: (I) Conception and design: G Pan, Y Fan; (II) Administrative support: Y Fan; (III) Provision of study materials or patients: Y Fan; \\ (IV) Collection and assembly of data: G Pan, K Chen, J Sheng; (V) Data analysis and interpretation: G Pan, K Chen, X Yu; (VI) Manuscript writing: \\ All authors; (VII) Final approval of manuscript: All authors. \\ Correspondence to: Yun Fan, MD. The First Clinical Medical College of Wenzhou Medical University, Ouhai District, Wenzhou, 325035 , China. \\ Email: fanyun@zjcc.org.cn.
}

\begin{abstract}
Background: Osimertinib has been adopted as the standard therapy for T790M-mediated acquired resistance to first-line epidermal growth factor receptor tyrosine kinase inhibitors (EGFR-TKIs) in patients with non-small cell lung cancer (NSCLC). The detection of EGFR T790M can be evaluated using different methods. The association between baseline T790M abundance and osimertinib efficacy has not been fully determined.

Methods: A total of 144 advanced NSCLC patients positive for T790M, at the time of progression, were retrospectively enrolled in this study. The effect of abundance of T790M mutation on the efficacy of osimertinib was explored.
\end{abstract}

Results: Among the 144 patients receiving T790M testing, 20 (13.9\%) had adopted amplification refractory mutation system (ARMS), 63 (43.8\%) adopted droplet digital PCR (ddPCR), and 61 (42.4\%) used next-generation sequencing (NGS). The objective response rate was $54.2 \%$, the median progressionfree survival was 12.0 months, and the overall survival was 23.0 months for the NSCLC patients treated with osimertinib. Three different technologies to assess T790M mutation (including ARMS, ddPCR, and NGS) could accurately predict the efficacy of osimertinib. There was no significant relationship between the abundance of T790M mutation and the efficacy of osimertinib.

Conclusions: ARMS, ddPCR, and NGS are reliable methods to evaluate EGFR T790M mutation. Osimertinib was equally effective for NSCLC patients with various abundance of T790M mutation.

Keywords: Non-small cell lung cancer (NSCLC); T790M abundance; droplet digital PCR (ddPCR); next generation sequencing (NGS); osimertinib

Submitted Feb 03, 2021. Accepted for publication Apr 14, 2021.

doi: $10.21037 /$ tcr-21-223

View this article at: https://dx.doi.org/10.21037/tcr-21-223

\section{Introductions}

Epidermal growth factor receptor (EGFR) mutations are observed in approximately $30-40 \%$ in Asian non-small cell lung cancer (NSCLC) patients compared with approximately $20 \%$ in Caucasians (1); typically deletions in exon 19 (EGFRdel19) and a point mutation in the exon 21 (EGFR L858R) (2). The first- and second-generation EGFR tyrosine kinase inhibitors (TKIs) such as gefitinib, erlotinib, and afatinib have shown significant effects for

\footnotetext{
$\wedge$ ORCID: 0000-0002-9757-235X.
} 
EGFR-mutant NSCLC patients, to stunt the growth of tumors with a median progression-free survival (PFS) of $10-14$ months $(3,4)$. However, cancer cells inevitably acquire resistance to EGFR-TKIs through different mechanisms, of which the EGFR T790M resistance mutation is reported in approximately $50-60 \%$ of the cases $(5,6)$. Osimertinib, a third-generation EGFR-TKI, has high activity against both EGFR T790M and classic EGFR mutations $(7,8)$. Osimertinib has been adopted as the standard care for T790M-mediated acquired resistance NSCLC patients $(9,10)$.

The assessment of T790M using tissue biopsy or plasma is mandatory at disease progression, after treatment with first-line EGFR-TKIs. Tissue biopsy is recommended first, although it is associated with challenges such as difficulty of invasive re-biopsy and tumor heterogeneity (11-13). The analysis of circulating cell-free tumor DNA (ctDNA) is an alternative approach that involves molecular analysis, where tumor biopsy is not feasible; it avoids the challenges above and enables real-time monitoring of the clonal evolution $(14,15)$. Currently, amplification refractory mutation system (ARMS), droplet digital PCR (ddPCR), and next-generation sequencing (NGS) are representative clinical platforms for T790M detection both in tissue and plasma (16). The impact of different technologies in assessing the T790M alteration and predicting the efficacy of osimertinib in a real-world setting has not been evaluated much. Meanwhile, studies have indicated potential associations between EGFR T790M abundance and efficacy of Osimertinib; however, the results are controversial (17-23). Therefore, the baseline abundance of T790M that serves as a predictive biomarker of response to osimertinib needs further identification.

This study compared the efficacy of osimertinib in T790M-positive patients with various technologies including ARMS, ddPCR, and NGS test, as well as analyzed the association between the baseline abundance of T790M and the efficacy of osimertinib in advanced NSCLC

We present the following article in accordance with the STROBE reporting checklist (available at https://dx.doi. org/10.21037/tcr-21-223).

\section{Methods}

\section{Participants and data collection}

Data from 157 T790M-positive advanced NSCLC patients, who received osimertinib treatment at the Zhejiang cancer hospital between April 2017 and December 2019, were retrospectively collected. Clinical data was obtained from the electronic medical record database, and the last followup was done in May 2020. The study was conducted in accordance with the Declaration of Helsinki (as revised in 2013). The study was approved by the Ethics Committee of Zhejiang Cancer Hospital, Hangzhou, China (NO. IRB2021-111), and informed consent was taken from all the patients.

\section{ARMS-PCR, ddPCR and NGS measurement}

ARMS assay was mostly performed using ABI 7500 (Applied Biosystems, Foster City, CA, USA), ddPCR assay was mostly performed using QX200 Droplet Digital PCR (BIO-RAD, Hercules, CA, USA) system, and NGS assay was mostly performed using MiSeqDX (Illumina, San Diego, CA, USA). Generally, the minimum detection limit of ddPCR is $0.01 \%$ when providing sufficient sample and operating according to the standard procedures. The highthroughput sequencing of NGS contained 168 genes related to the pathogenesis and targeted therapy with $\geq 500$ average sequencing depth, and the detection for alterations covered single-nucleotide variant (SNV), short fragment insertions or deletions (INDEL), copy number variation (CNV) and rearrangements within the range of $+/-20$ bp of target gene exon. The T790M abundance was calculated as mutant allele frequency (MAF), which indicated the fraction of mutated alleles relative to the corresponding WT allele to analyze the allele fractions of T790M.

\section{Assessment of efficacy}

Tumor response was examined using computed tomography and was evaluated according to the Response Evaluation Criteria in Solid Tumors (RECIST) version 1.1. The objective response rate (ORR) was defined as the percentage of patients with complete or partial response (CR or PR). The progression-free survival PFS was defined as the time from the first day of osimertinib treatment to tumor progression or death. The overall survival (OS) was defined as the time from Osimertinib treatment to death of any cause. Radiologic assessments for survival were performed approximately every 6-8 weeks until objective disease progression or loss of follow-up.

\section{Statistical analysis}

The PFS or OS was estimated using the Kaplan-Meier method and then compared using the log-rank test. The 
baseline characteristics, ORR and relationship between T790M mutation abundance were compared using the Pearson's chi-square test, Mann-Whitney test, or Fisher's exact test. Two-sided $\mathrm{P}$ values $<0.05$ were considered statistically significant. Statistical analyses and graphical representations were performed using SPSS version 23.0 for Windows (Chicago, IL, USA) and GraphPad Prism (version 8.0) software.

\section{Results}

\section{Patient characteristics}

Thirteen patients were excluded due to the absence of information about the detection methods; 144 patients were enrolled. In total, 81 females and 63 males with a median age of 60 years (range, 36-81 years) were evaluated; $43(29.9 \%)$ patients have confirmed brain metastases; $88(61.1 \%)$ patients originally harbored EGFR exon 19 deletion and 52 (36.1\%) harbored L858R mutation. There were 140 patients (97.2\%) identified as harboring T790M mutation with EGFR-TKIs resistance, and four patients harboring de novo EGFR T790M mutation were also included. Prior EGFR-TKIs were gefitinib in 52 (36.1\%) patients, icotinib in $85(59.0 \%)$ patients, erlotinib in $2(1.4 \%)$ patients and afatinib in $1(0.7 \%)$ patient. Most of the patients received osimertinib directly or after chemotherapy when resistance to prior EGFR-TKIs, and no records of patients receiving second-generation EGFR-TKI after resistance to gefitinib or icotinib. The median follow-up time was 24.5 months. The baseline characteristics of the patients are presented in Table 1.

\section{Clinical outcomes of osimertinib in real-world}

A total of 144 patients were evaluated for response to osimertinib and had an ORR of $54.2 \%$. The median PFS was 12.0 months (95\% CI: 9.8-14.2 months; Figure 1A), and the median OS was 23.0 months (95\% CI: 16.2-29.8 months; Figure 1B). The ORR of patients with brain metastases $(n=43)$ was slightly lower than those without ( $51.2 \%$ vs. $56.7 \%$; $\mathrm{P}=0.584$; Figure $1 C$ ), although the difference was not significant. Similarly, both the median PFS and OS for patients with brain metastases was shorter than patients without brain metastases; although the difference was not statistically significant (mPFS: 10.0 vs. 14.0 months; $\mathrm{P}=0.145$; mOS: 16.0 vs. 27.0 months; $\mathrm{P}=0.170$; Figure $1 D, E)$.

\section{Comparison of osimertinib efficacy between plasma detection and tissue biopsy}

A total of 112 patients with EGFR T790M were detected by peripheral blood while 28 patients by tissue biopsy respectively. The ORR of patients detected by plasma and tissue were $53.6 \%$ and $60.7 \%$ respectively, and the difference was not statistically significant $(\mathrm{P}=0.301$; Figure $2 A$ ). Moreover, survival analysis showed that there was no significant difference in median PFS and median OS of patients detected by plasma and tissue (plasma $v$ s. tissue; mPFS: 12.0 vs. 14.0 months, $\mathrm{P}=0.209$; $\mathrm{mOS}: 23.0$ vs. 22.0 months, $\mathrm{P}=0.207$; Figure $2 B, C$ ).

\section{Comparison of different detecting methods on T790M mutation}

Among the 144 patients, 20 (13.9\%) had adopted ARMS, $63(43.8 \%)$ adopted ddPCR, and 61 (42.4\%) used NGS in T790M testing. Most of the sample (140/144=97.2\%) types were tissue or peripheral blood; however, 2 cases of pleural effusion (one detected using ARMS and the other using NGS) and 2 cases of cerebrospinal fluid (both detected by NGS) were also included. Although T790M detection was performed using three methods, there was no significant difference on the efficacy of osimertinib in ORR (ARMS vs. ddPCR vs. NGS: $65 \%$ vs. $49.2 \%$ vs. $55.7 \%$, respectively; $\mathrm{P}=0.443$; Figure $3 A$ ), median PFS (14.0 vs. 12.6 vs. 14.0 months; respectively, log-rank $\mathrm{P}=0.415$; Figure $3 B$ ) and median OS (23.0 vs. 19.0 vs. 27.0 months; respectively, logrank $\mathrm{P}=0.459$; Figure 3 ).

\section{Relationship between baseline abundance of T790M mutation and the efficacy of Osimertinib}

In general, data of T790M-mutant abundance were obtained in 77 patients (53.5\%); 57 were tested using ddPCR and the last 20 using NGS. The median abundance of T790M mutation was $1.41 \%$ (range, $0.02-49.74 \%$ ) among the 77 patients. We first compared the median T790M-mutant abundance level between responders and non-responders. The median abundance for the two groups was $1.26 \%$ (range, $0.03 \%$ to $47.81 \%$ ) and $1.46 \%$ (range, $0.02 \%$ to $49.74 \%$ ), respectively; there was no significant difference $(\mathrm{P}=0.966$; Figure $4 A)$. Since we did not find the best cutoff value of T790M abundance predicting objective response (Figure $4 B$ ), we next divided the patients into two groups, the low-abundance $(\mathrm{n}=39)$ and high-abundance 
Table 1 Baseline characteristics of patients

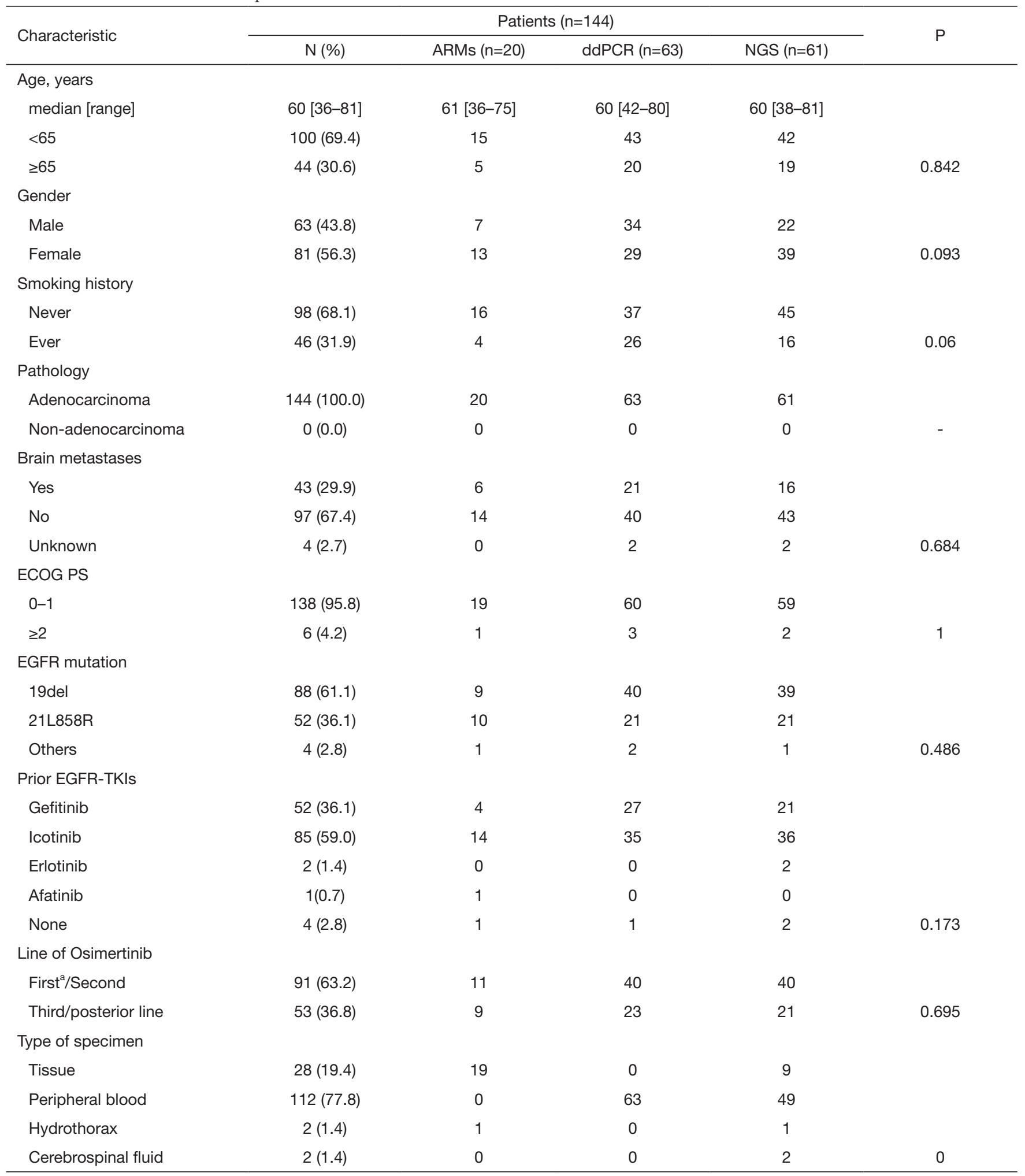

${ }^{a}$, Four patients had de novo T790M mutation and osimertinib were the first-line therapy. ECOG, Eastern Cooperative Oncology Group; PS, performance status; ARMS, amplification refractory mutation system; ddPCR, droplet digital PCR; NGS next-generation sequencing; EGFR, epidermal growth factor receptor; TKIs, tyrosine kinase inhibitors. 

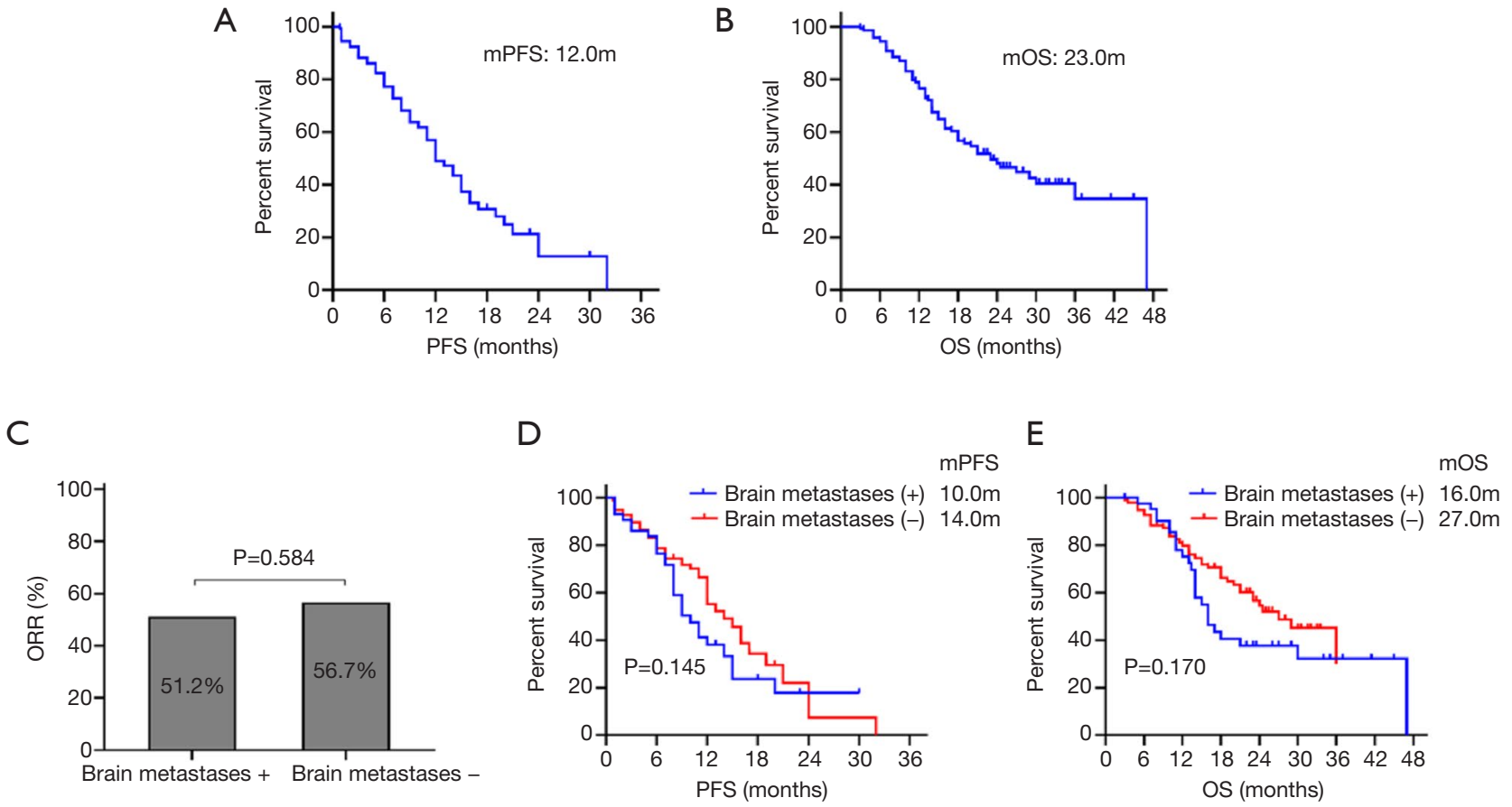

Figure 1 Clinical outcomes of osimertinib in real-world. (A) Progression-free survival (PFS) in all patients. (B) The overall survival (OS) in all patients. (C) Response to osimertinib in patients with or without brain metastases. (D) The PFS of patients stratified by brain metastasis. (E) The OS of patients stratified by brain metastasis.
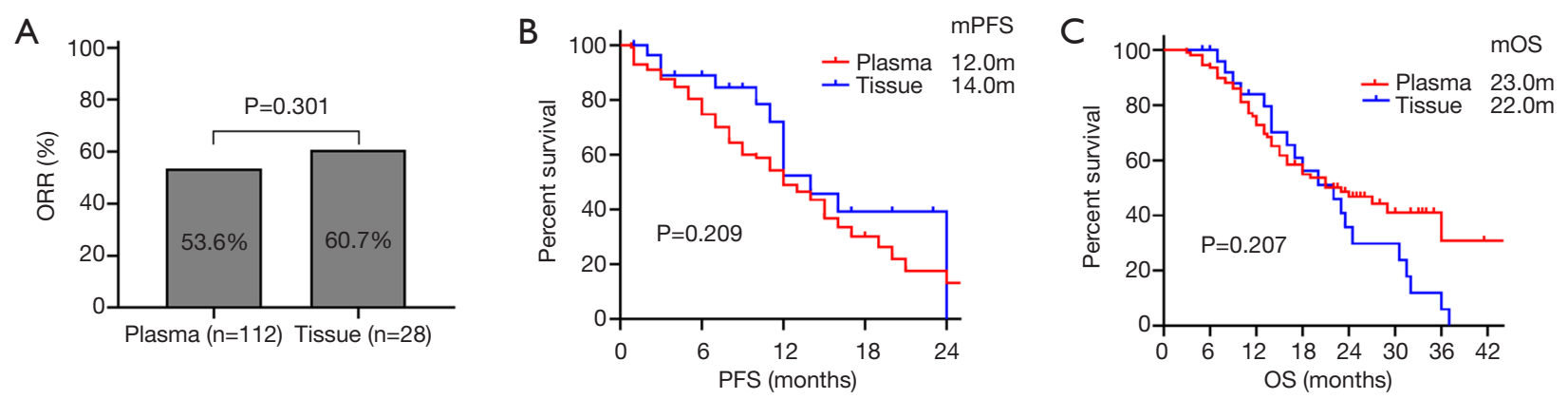

Figure 2 Comparison of osimertinib efficacy between plasma detection and tissue biopsy. (A) Comparison of response to osimertinib of patients detected by plasma and tissue. (B) The progression-free survival (PFS) of patients stratified by samples. (C) The overall survival (OS) of patients stratified by samples.

$(\mathrm{n}=38)$, according to the median abundance of T790M mutation. The baseline characteristics of the two groups in age, sex, smoking history, brain metastases, ECOG PS, EGFR mutation, prior EGFR-TKIs and line of Osimertinib usage were all comparable (Table 2). However, there was no significant difference in ORR, median PFS and median OS between the two groups (Low-abundance $v s$. Highabundance; ORR: $51.3 \%$ vs. $47.4 \%$; $\mathrm{P}=0.731$; mPFS: 12.0 vs. 12.0 mouths; $\mathrm{P}=0.800$; mOS: 21.0 vs. 18.0 mouths; $\mathrm{P}=0.502$; Figure $4 C, D, E)$.

\section{Subgroup analysis of the association between T790M-mutant abundance and osimertinib outcomes}

The median abundance for ddPCR and NGS groups were $0.94 \%$ (range, $0.02 \%$ to $49.74 \%$ ) and $6.26 \%$ (range, $0.03 \%$ 

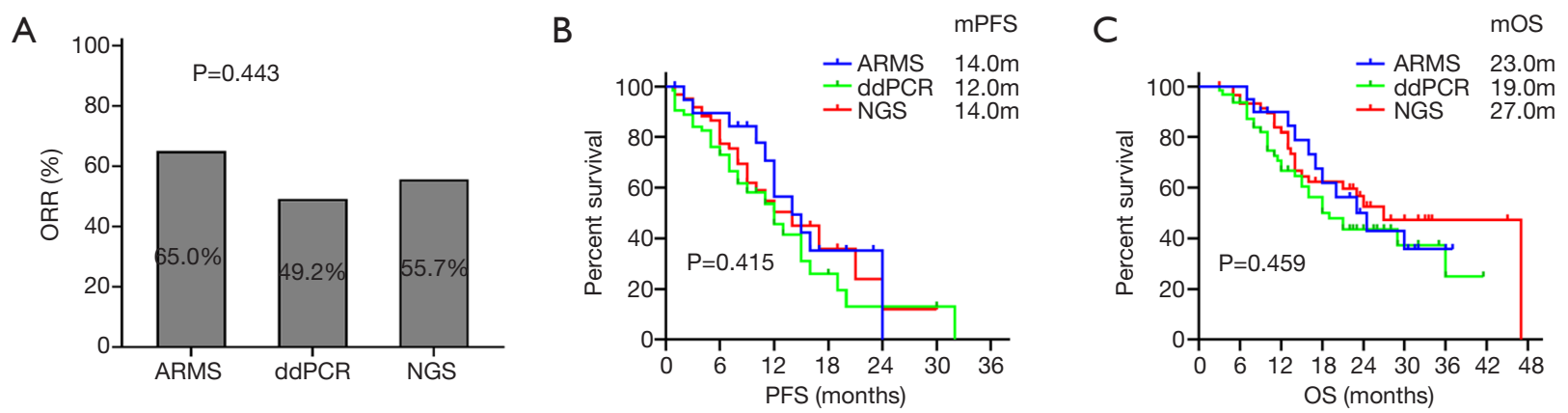

Figure 3 Comparison of different detecting methods on T790M mutation. (A) Comparison of response to osimertinib across the amplification refractory mutation system (ARMS), droplet digital PCR (ddPCR), next-generation sequencing (NGS) groups. (B) The progression-free survival (PFS) of patients stratified by detection platforms. (C) The overall survival (OS) of patients stratified by detection platforms.
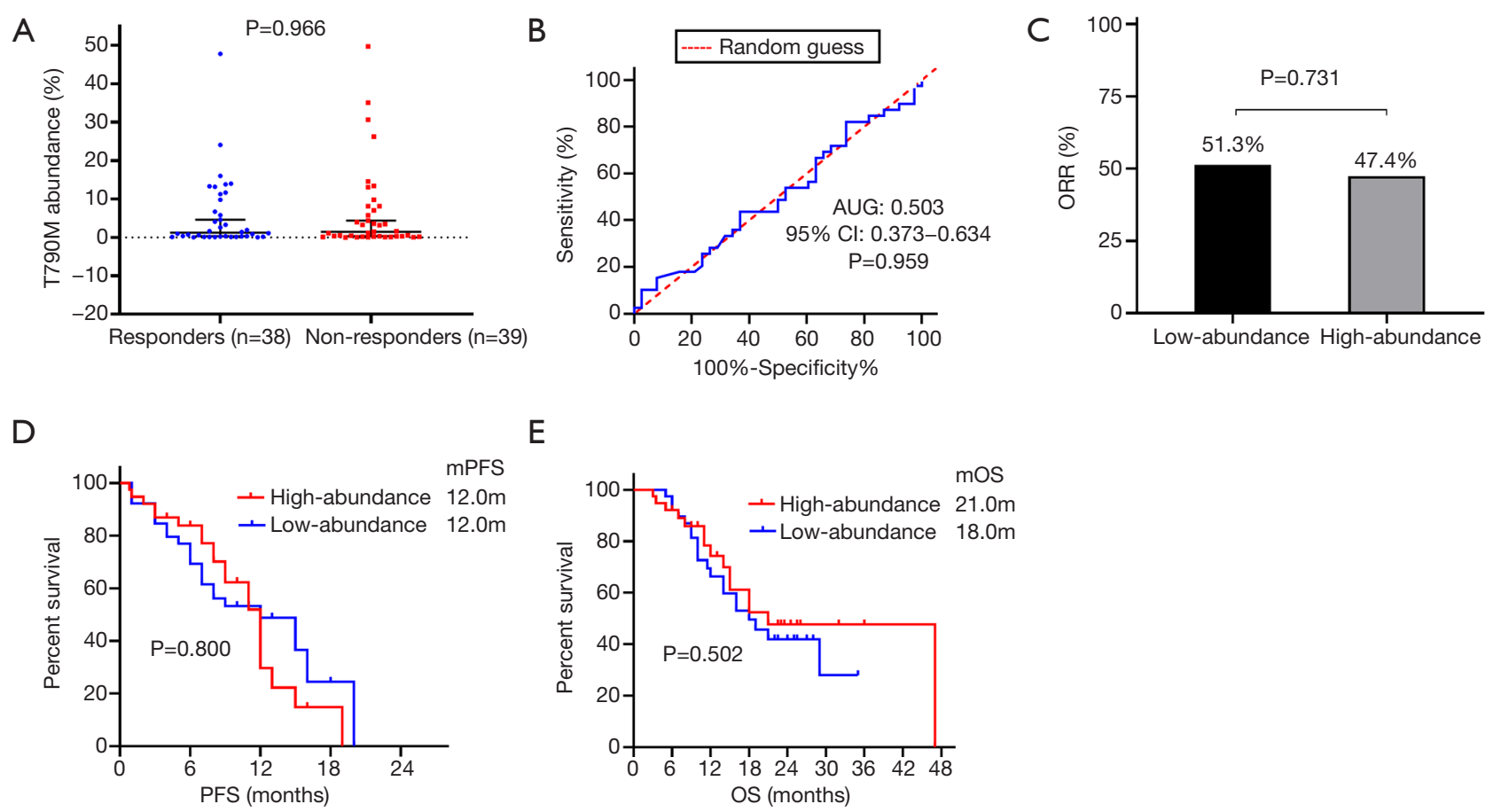

Figure 4 Association between T790M-mutant abundance and osimertinib outcomes. (A) T790M-mutant abundance between responders and non-responders (Mann-Whitney test). (B) Receiver operating characteristic (ROC) Curve for T790M abundance predicting objective response. (C) Overall response rate between patients with low-abundance and high-abundance $\left(\chi^{2}\right.$ test). (D) Progression-free survival (PFS) between patients with low-abundance and high-abundance (log-rank test). (E) Overall survival (OS) between patients with low-abundance and high-abundance (log-rank test).

to $47.81 \%)$, respectively ( $\mathrm{P}=0.022$; Figure $5 A)$. There was no significant difference between responders and nonresponders of median T790M-mutant abundance in both ddPCR and NGS groups (responders vs. non-responders; ddPCR: $0.91 \%$ vs. $1.09 \%, \mathrm{P}=0.877$; NGS: $8.96 \%$ vs. $4.86 \%$,
$\mathrm{P}=0.343$; Figure 5B). The patients were then divided into the low- and high-abundance groups according to the median abundance of T790M mutation; there was no significant difference found in the ORR for both ddPCR and NGS (ddPCR: $55.20 \%$ vs. $50.0 \%, \mathrm{P}=0.696$; NGS: $30.0 \%$ vs. 
Table 2 Baseline characteristics of patients with data of T790M-mutant abundance

\begin{tabular}{|c|c|c|c|}
\hline Characteristic & \multicolumn{2}{|c|}{ Patients $(n=77)$} & $P$ value \\
\hline \multicolumn{4}{|l|}{ Age, years } \\
\hline Median [range] & 63 [38-80] & 58 [42-74] & \\
\hline$<60$ & 15 & 22 & 0.139 \\
\hline \multicolumn{4}{|l|}{ Gender } \\
\hline Male & 19 & 20 & \\
\hline Female & 20 & 18 & 0.731 \\
\hline \multicolumn{4}{|l|}{ Smoking history } \\
\hline \multicolumn{4}{|l|}{ Brain metastases } \\
\hline Yes & 13 & 9 & \\
\hline No & 24 & 27 & \\
\hline Unknown & 2 & 2 & 0.491 \\
\hline \multicolumn{4}{|l|}{ ECOG PS } \\
\hline $0-1$ & 38 & 38 & \\
\hline$\geq 2$ & 1 & 0 & 1.000 \\
\hline \multicolumn{4}{|l|}{ EGFR mutation } \\
\hline Icotinib & 23 & 19 & \\
\hline Others/None & 0 & 3 & 0.272 \\
\hline \multicolumn{4}{|c|}{ Line of osimertinib usage } \\
\hline First ${ }^{\mathrm{a}} /$ Second & 23 & 30 & \\
\hline Third/posterior line & 16 & 8 & 0.100 \\
\hline
\end{tabular}

The baseline characteristics were compared using the Pearson's chi-square test or Fisher's exact test. ECOG, Eastern Cooperative Oncology Group; PS, performance status; ARMS, amplification refractory mutation system; ddPCR, droplet digital PCR; NGS nextgeneration sequencing; EGFR, epidermal growth factor receptor; TKIs, tyrosine kinase inhibitors.

$50.0 \%, \mathrm{P}=0.650$; Figure 5C,D). Additionally, there was similar PFS and OS in the low- and high-abundance groups tested using ddPCR (mPFS: 15.0 mouths vs. 12.0 mouths;
$\mathrm{P}=0.409$; mOS: 19.0 vs. 18.0 mouths; $\mathrm{P}=0.670$; Figure $5 E, F$ ), as well as NGS group (mPFS: 7.0 vs. 9.0 mouths; $\mathrm{P}=0.154$; mOS: 14.0 vs. 47.0 mouths; $\mathrm{P}=0.141$; Figure $5 G, H)$. 


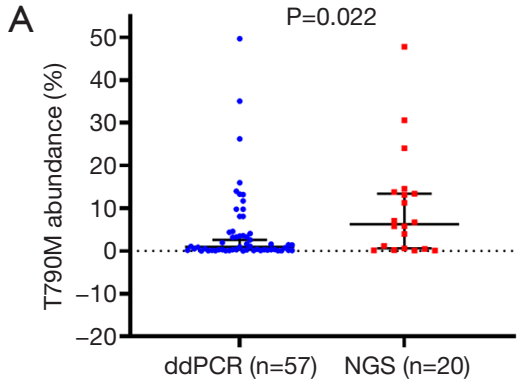

C

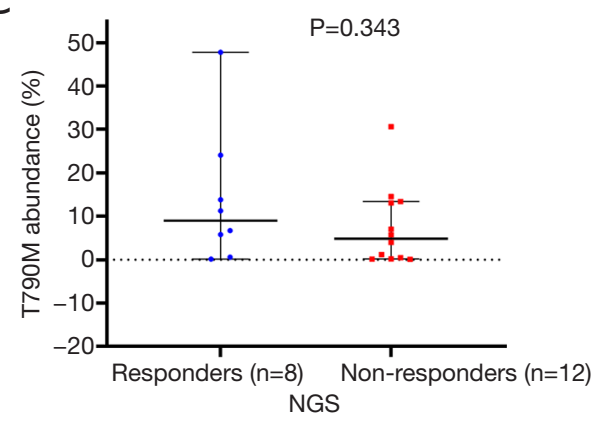

E

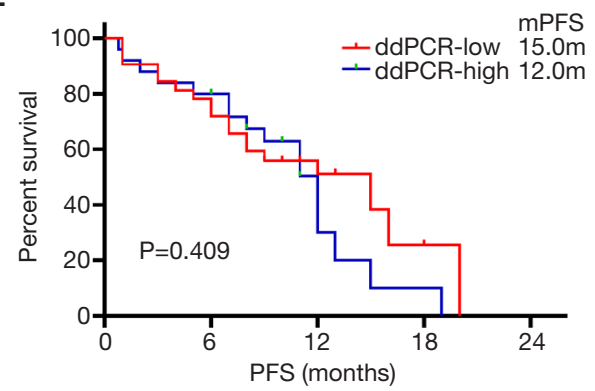

G

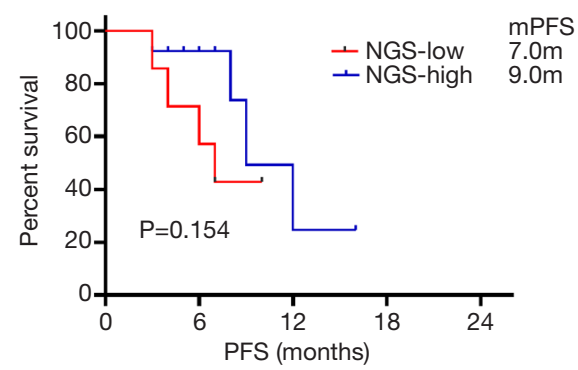

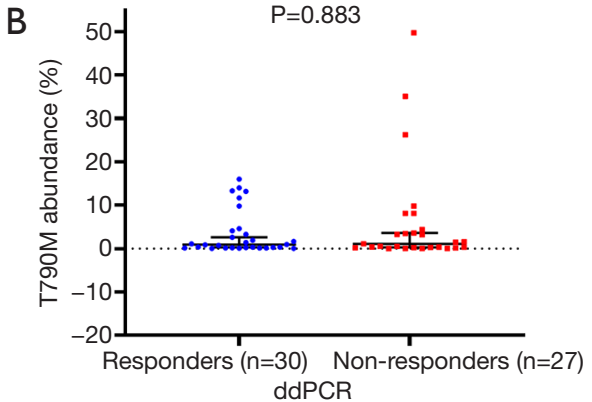
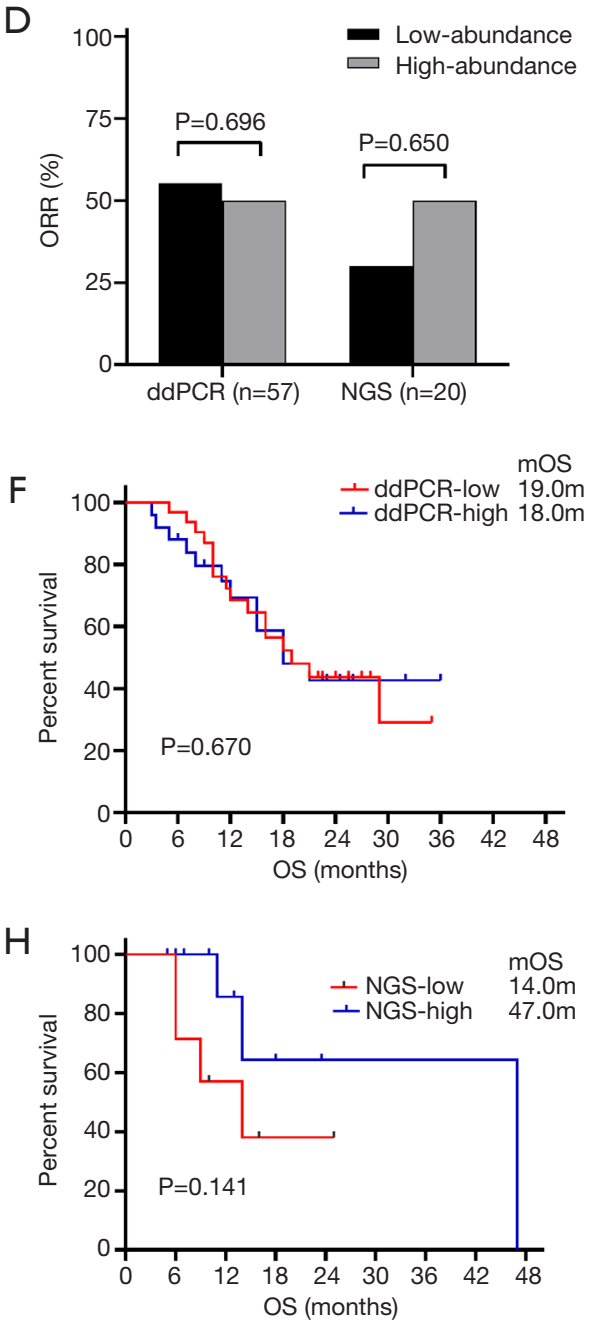

Figure 5 Subgroup analysis of the association between T790M-mutant abundance and osimertinib outcomes. (A) T790M-mutant abundance in groups of droplet digital PCR (ddPCR) and generation sequencing (NGS). (B) T790M-mutant abundance between responders and non-responders tested using ddPCR (Mann-Whitney test). (C) T790M-mutant abundance between responders and non-responders tested using NGS (Mann-Whitney test). (D) The overall response rate between patients with low-abundance and high-abundance $\left(\chi^{2}\right.$ test and Fisher's exact test). (E) The progression-free survival (PFS) stratified into the low-abundance and high-abundance in ddPCR group (log-rank test). (F) Overall survival (OS) stratified into the low-abundance and high-abundance in ddPCR groups (log-rank test). (G) The PFS stratified into the low-abundance and high-abundance in NGS group (log-rank test). (H) OS stratified by low-abundance and highabundance in NGS groups (log-rank test). 


\section{Discussion}

The detection sensitivity of T790M using ARMS, ddPCR, and NGS tests were found to be useful and reliable in clinical practice. Moreover, osimertinib was equally effective for NSCLC patients with various levels of T790M mutation abundance with resistance to first- and second-generation EGFR-TKIs.

Among 144 patients with T790M-positive NSCLC, the ORR to osimertinib was $54.2 \%$, median PFS was 12.0 months, and median OS was 23.0 months. These results were equivalent to the data in a phase 3 clinical trial, which reported an mPFS of 10.1 months and mOS of 26.8 months $(9,10)$. The curative effect of patients with brain metastases was inferior to those without brain metastases (mPFS: $10.0 \mathrm{vs.}$ 14.0 months; $\mathrm{P}=0.145$; mOS: 16.0 vs. 27.0 months; $\mathrm{P}=0.170$ ), although the difference was not statistically significant. Therefore, osimertinib, a third-generation EGFR-TKI, is strongly active against T790M-positive NSCLC.

Furthermore, there were similar treatment outcomes for osimertinib in the T790M-mutant population selected through the above three detected methods, although there were several differences among these approaches in sensitivity, specificity, and sample selection. The ARMS was a widely used method for T790M mutation testing with good specificity, however, it was limited in liquid biopsy because it lacked sensitivity when compared to ddPCR and NGS (16). A cross-platform comparison reported higher sensitivity (71\%) and concordance (74\%) of ddPCR in detecting T790M mutation between plasma ctDNA and tissue, compared to ARMS with sensitivity of $21 \%$ and concordance of $48 \%$ (24). Another study demonstrated the high sensitivity (81.8\%) and good concordance (86\%) of NGS in the detection of T790M mutation in plasma ctDNA when compared to tumor tissues (25). Collectively, the quantification platforms such as ddPCR and NGS were superior to ARMS in T790M mutation detection, particularly in liquid biopsy such as plasma ctDNA (16).

In this study, the samples tested using ddPCR were all plasma ctDNA, whereas those tested using ARMS were nearly all tissue (one case was pleural effusion), and more than half of the samples tested by NGS were plasma ctDNA. Choosing an appropriate sample and method could significantly improve the detection accuracy of T790M. Also, definite effects of osimertinib were observed for T790M-positive patients who were identified to have T790M mutations by analyzing ctDNA in pleural effusion and cerebrospinal fluid. Though there are limited studies on the use of pleural effusion and cerebrospinal fluid in T790M mutation detection in lung cancer, the results suggested that they were clinically available bio-samples which may better reflect cells from the entire tumor and help predict treatment response and prognosis.

The findings from the analysis of the relationship between the proportion of EGFR T790M mutation and the response to osimertinib treatment have been controversial (17-23). Studies have found that a higher ratio of the allele fraction of T790M to mutated EGFR (T790M/act-EGFR MAF) in plasma or tissues was associated with a significantly better efficacy of Osimertinib (17-19,21), whereas another study reported that a higher allele fraction of T790M in circulating tumor DNA were associated with a poor response and shorter PFS to osimertinib treatment $(20,22)$. Regrettably, our study could not find an exact relationship between the baseline abundance of T790M mutation and the efficacy of osimertinib.

There are several reasons that may account for these contradictions. First, the heterogeneity of resistance mechanisms within tumors may have affected our results. Clones lacking the T790M mutation are likely to have other resistance mechanisms such as MET amplification, ERBB2 amplification, PIK3CA mutation, transformation to smallcell lung cancer, and epithelial-to-mesenchymal transition $(6,26)$; such clones would not respond to osimertinib. Secondly, T790M mutation abundance may not be the best predictive biomarker. The T790M mutation abundance was calculated as mutant allele frequency (MAF) in this study. However, increasing evidence has shown that EGFRmutant NSCLC is not a single-oncogene disease $(27,28)$, and both EGFR-mutated and wild-type cancer cells exist simultaneously (29-31). Zheng et al. (23) proposed a concept of "the T790M relative mutation purity (RMP)" as the ratio of $\mathrm{T} 790 \mathrm{M}$ allele frequency $(\mathrm{AF})$ to maximum somatic allele frequency, and it may be an efficient biomarker to predict the efficacy of osimertinib, which need further confirmation. Thirdly, the sensitivity of the plasma ctDNA test is higher in patients with extra-thoracic metastases and multiple metastatic sites, which reflect a higher overall tumor burden (20). High T790M mutant copy numbers may reflect higher tumor loads and worse performance status, which is intrinsically associated with poor prognosis. Therefore, limited benefits have been observed in the patient with a high T790M mutation abundance.

This study has several limitations. First, data of T790M mutation detection were mostly obtained from the electronic medical record database; therefore the detection processes were not highly standardized and uniform. 
Secondly, tissue samples, peripheral blood, pleural effusion, and cerebrospinal fluid were all included, though evidence had demonstrated that some clinically available biosamples such pleural effusion and cerebrospinal fluid were able to serve as an alternative for tissue and blood. Third, the retrospective nature of the analysis may yield bias. Therefore, future prospective studies will be necessary to strengthen these results.

In conclusion, the results suggest that ARMS, ddPCR, and NGS are clinically effective and optional approaches for T790M mutation detection. Furthermore, the efficacy of osimertinib was similar among NSCLC patients with different abundance of T790M mutation.

\section{Acknowledgments}

We would like to acknowledge Dr. Jeremiah Machuki for assistance in formatting and language editing.

Funding: None.

\section{Footnote}

Reporting Checklist: The authors have completed the STROBE reporting checklist. Available at https://dx.doi. org/10.21037/tcr-21-223

Data Sharing Statement: Available at https://dx.doi. org/10.21037/tcr-21-223

Peer Review File: Available at https://dx.doi.org/10.21037/ tcr-21-223

Conflicts of Interest: All authors have completed the ICMJE uniform disclosure form (available at https://dx.doi. org/10.21037/tcr-21-223). The authors have no conflicts of interest to declare.

Ethical Statement: The authors are accountable for all aspects of the work in ensuring that questions related to the accuracy or integrity of any part of the work are appropriately investigated and resolved. The study was conducted in accordance with the Declaration of Helsinki (as revised in 2013). The study was approved by the Ethics Committee of Zhejiang Cancer Hospital, Hangzhou, China (NO. IRB-2021-111), and informed consent was taken from all the patients.

Open Access Statement: This is an Open Access article distributed in accordance with the Creative Commons Attribution-NonCommercial-NoDerivs 4.0 International License (CC BY-NC-ND 4.0), which permits the noncommercial replication and distribution of the article with the strict proviso that no changes or edits are made and the original work is properly cited (including links to both the formal publication through the relevant DOI and the license). See: https://creativecommons.org/licenses/by-nc-nd/4.0/.

\section{References}

1. Zhang YL, Yuan JQ, Wang KF, et al. The prevalence of EGFR mutation in patients with non-small cell lung cancer: a systematic review and meta-analysis. Oncotarget 2016;7:78985-93.

2. Wang J, Ramakrishnan R, Tang Z, et al. Quantifying EGFR alterations in the lung cancer genome with nanofluidic digital PCR arrays. Clin Chem 2010;56:623-32.

3. Maemondo M, Inoue A, Kobayashi K, et al. Gefitinib or chemotherapy for non-small-cell lung cancer with mutated EGFR. N Engl J Med 2010;362:2380-8.

4. Rosell R, Carcereny E, Gervais R, et al. Erlotinib versus standard chemotherapy as first-line treatment for European patients with advanced EGFR mutation-positive non-small-cell lung cancer (EURTAC): a multicentre, open-label, randomised phase 3 trial. Lancet Oncol 2012;13:239-46.

5. Kuiper JL, Heideman DA, Thunnissen E, et al. Incidence of T790M mutation in (sequential) rebiopsies in EGFRmutated NSCLC-patients. Lung Cancer 2014;85:19-24.

6. Yu HA, Arcila ME, Rekhtman N, et al. Analysis of tumor specimens at the time of acquired resistance to EGFR-TKI therapy in 155 patients with EGFR-mutant lung cancers. Clin Cancer Res 2013;19:2240-7.

7. Cross DA, Ashton SE, Ghiorghiu S, et al. AZD9291, an irreversible EGFR TKI, overcomes T790M-mediated resistance to EGFR inhibitors in lung cancer. Cancer Discov 2014;4:1046-61.

8. Jänne PA, Yang JC, Kim DW, et al. AZD9291 in EGFR inhibitor-resistant non-small-cell lung cancer. $\mathrm{N}$ Engl J Med 2015;372:1689-99.

9. Mok TS, Wu YL, Ahn MJ, et al. Osimertinib or PlatinumPemetrexed in EGFR T790M-Positive Lung Cancer. N Engl J Med 2017;376:629-40.

10. Papadimitrakopoulou VA, Mok TS, Han JY, et al. Osimertinib versus platinum-pemetrexed for patients with EGFR T790M advanced NSCLC and progression on a prior EGFR-tyrosine kinase inhibitor: AURA3 overall 
survival analysis Ann Oncol 2020;31:1536-44.

11. Fisher R, Pusztai L, Swanton C. Cancer heterogeneity: implications for targeted therapeutics. Br J Cancer 2013;108:479-85.

12. Tokaca N, Barth S, O'Brien M, et al. Molecular Adequacy of Image-Guided Rebiopsies for Molecular Retesting in Advanced Non-Small Cell Lung Cancer: A Single-Center Experience. J Thorac Oncol 2018;13:63-72.

13. Weber B, Meldgaard P, Hager H, et al. Detection of EGFR mutations in plasma and biopsies from non-small cell lung cancer patients by allele-specific PCR assays. BMC Cancer 2014;14:294.

14. Diehl F, Schmidt K, Choti MA, et al. Circulating mutant DNA to assess tumor dynamics. Nat Med 2008;14:985-90.

15. Douillard JY, Ostoros G, Cobo M, et al. Gefitinib treatment in EGFR mutated caucasian NSCLC: circulating-free tumor DNA as a surrogate for determination of EGFR status. J Thorac Oncol 2014;9:1345-53.

16. Li X, Zhou C. Comparison of cross-platform technologies for EGFR T790M testing in patients with non-small cell lung cancer. Oncotarget 2017;8:100801-18.

17. Ariyasu R, Nishikawa S, Uchibori K, et al. High ratio of T790M to EGFR activating mutations correlate with the osimertinib response in non-small-cell lung cancer. Lung Cancer 2018;117:1-6.

18. Del Re M, Bordi P, Rofi E, et al. The amount of activating EGFR mutations in circulating cell-free DNA is a marker to monitor osimertinib response. Br J Cancer 2018;119:1252-8.

19. Kuo CS, Huang CH, Liu CY, et al. Prior EGFR-TKI Treatment in EGFR-Mutated NSCLC Affects the Allele Frequency Fraction of Acquired T790M and the Subsequent Efficacy of Osimertinib. Target Oncol 2019;14:433-40.

20. Li JY, Ho JC, Wong KH. T790M mutant copy number quantified via ddPCR predicts outcome after osimertinib treatment in lung cancer. Oncotarget 2018;9:27929-39.

21. Oxnard GR, Thress KS, Alden RS, et al. Association Between Plasma Genotyping and Outcomes of Treatment With Osimertinib (AZD9291) in Advanced Non-Small-

Cite this article as: Pan G, Chen K, Yu X, Sheng J, Fan Y. The correlation between the abundance of EGFR T790M mutation and osimertinib response in advanced non-small cell lung cancer. Transl Cancer Res 2021;10(6):2895-2905. doi: $10.21037 /$ tcr-21-223
Cell Lung Cancer. J Clin Oncol 2016;34:3375-82.

22. Remon J, Caramella C, Jovelet C, et al. Osimertinib benefit in EGFR-mutant NSCLC patients with T790Mmutation detected by circulating tumour DNA. Ann Oncol 2017;28:784-90.

23. Zheng Q, Hong S, Huang Y, et al. EGFR T790M relative mutation purity predicts osimertinib treatment efficacy in non-small cell lung cancer patients. Clin Transl Med 2020;9:17.

24. Thress KS, Brant R, Carr TH, et al. EGFR mutation detection in ctDNA from NSCLC patient plasma: A crossplatform comparison of leading technologies to support the clinical development of AZD9291. Lung Cancer 2015;90:509-15.

25. Paweletz CP, Sacher AG, Raymond CK, et al. BiasCorrected Targeted Next-Generation Sequencing for Rapid, Multiplexed Detection of Actionable Alterations in Cell-Free DNA from Advanced Lung Cancer Patients. Clin Cancer Res 2016;22:915-22.

26. Gay L, Baker AM, Graham TA. Tumour Cell Heterogeneity. F1000Res 2016;5:F1000 Faculty Rev-238.

27. Blakely CM, Watkins TBK, Wu W, et al. Evolution and clinical impact of co-occurring genetic alterations in advanced-stage EGFR-mutant lung cancers. Nat Genet 2017;49:1693-704.

28. Hong S, Gao F, Fu S, et al. Concomitant Genetic Alterations With Response to Treatment and Epidermal Growth Factor Receptor Tyrosine Kinase Inhibitors in Patients With EGFR-Mutant Advanced Non-Small Cell Lung Cancer. JAMA Oncol 2018;4:739-42.

29. Taniguchi K, Okami J, Kodama K, et al. Intratumor heterogeneity of epidermal growth factor receptor mutations in lung cancer and its correlation to the response to gefitinib. Cancer Sci 2008;99:929-35.

30. Sakurada A, Lara-Guerra H, Liu N, et al. Tissue heterogeneity of EGFR mutation in lung adenocarcinoma. J Thorac Oncol 2008;3:527-9.

31. Bai H, Wang Z, Wang Y, et al. Detection and clinical significance of intratumoral EGFR mutational heterogeneity in Chinese patients with advanced non-small cell lung cancer. PLoS One 2013;8:e54170. 